\title{
ON SOME I-CONVERGENT DOUBLE SEQUENCE SPACES OF FUZZY REAL NUMBERS DEFINED BY MODULUS FUNCTION
}

\begin{abstract}
In this article our aim to introduce some new I-convergent double sequence spaces of fuzzy real numbers defined by modulus function and studies their some topological and algebraic properties. Also we establish some inclusion relations.

KEY WORDS: fuzzy real number, $I$-convergence, double sequence, modulus function.
\end{abstract}

AMS Mathematics Subject Classification: 40A05, 40D25, 46A45, $46 \mathrm{E} 30$.

\section{Introduction}

The notion of fuzzy sets was introduced by Zadeh [16]. After that many authors have studied and generalized this notion in many ways, due to the potential of the introduced notion. Also it has wide range of applications in almost all the branches of studied in particular science, where mathematics is used. It attracted many workers to introduce different types of fuzzy sequence spaces.

Bounded and convergent sequences of fuzzy numbers were studied by Matloka [8]. Later on sequences of fuzzy numbers have been studied by Kaleva and Seikkala [2], Tripathy and Sarma ([13], [14]) and many others.

I-convergence of real valued sequence was studied at the initial stage by Kostyrko, Šalát and Wilczyński [4] which generalizes and unifies different notions of convergence of sequences. The notion was further studied by Šalát, Tripathy and Ziman [9].

Let $X$ be a non-empty set, then a non-void class $\subseteq 2^{X}$ (power set of $X$ ) is called an ideal if $I$ is additive (i.e. $A, B \in I \Rightarrow A \cup B i n I$ ) and hereditary (i.e. $A \in I$ and $B \cup A \Rightarrow B i n I$ ). An ideal $I \subseteq 2^{X}$ is said to be non-trivial if $I \neq 2^{X}$. A non-trivial ideal $I$ is said to be admissible if $I$ contains every 
finite subset of $N$. A non-trivial ideal $I$ is said to be maximal if there does not exist any non-trivial ideal $J \neq I$ containing $I$ as a subset.

Let $X$ be a non-empty set, then a non-void class $F \subseteq 2^{X}$ is said to be a filter in $X$ if $\phi \notin F, A, B \in F \Rightarrow A \cap B \in F$ and $A \in F, A \subseteq B \Rightarrow B \in F$. For any ideal $I$, there is a filter $\Psi(I)$ corresponding to $I$, given by

$$
\Psi(I)=\{K \subseteq N: N \backslash K \in I\}
$$

A modulus function $f$ is a function from $[0, \infty)$ to $[0, \infty)$ such that:

(i) $f(x)=0$ iff $x=0$

(ii) $f(x+y) \leq f(x)+f(y)$ for all $x, y \geq 0$,

(iii) $f$ is increasing,

(iv) $f$ is continuous from the right at 0 .

It follows that $f$ must be continous everywhere on $[0, \infty)$ and a modulus function may be bounded or not bounded.

Let $X$ be a linear metric space. A function is called paranorm if:

(1) $p(x) \geq 0$ for all $x \in X$

(2) $p(-x)=p(x)$ for all $x \in X$

(3) $p(x+y) \leq p(x)+p(y)$ for all $x, y \in X$,

(4) If $\left(\lambda_{n}\right)$ be a sequence of scalars such that as $\lambda_{n} \rightarrow 0$ and $\left(x_{n}\right)$ be a sequence of vectors with $p\left(x_{n}-x\right) \rightarrow 0$ as $n \rightarrow \infty$, then $p\left(\lambda_{n} x_{n}-\lambda x\right) \rightarrow 0$ as $n \rightarrow \infty$.

A paranorm $p$ for which $p(x)=0 \Rightarrow x=0$ is called total paranorm and the pair $(X, p)$ is called a total paranormed space.

\section{Definitions and background}

Let $D$ denote the set of all closed and bounded intervals $X=\left[a_{1}, b_{1}\right]$ on the real line $R$. For $X=\left[a_{1}, b_{1}\right] \in D$ and $Y=\left[a_{2}, b_{2}\right] \in D$, define $d(X, Y)$ by

$$
d(X, Y)=\max \left(\left|a_{1}-b_{1}\right|,\left|a_{2}-b_{2}\right|\right) .
$$

It is known that $(D, d)$ is a complete metric space.

A fuzzy real number $X$ is a fuzzy set on $R$ i.e. a mapping $X: R \rightarrow L(=$ $[0,1])$ associating each real number $t$ with its grade of membership $X(t)$.

The $\alpha$-level set $[X]^{\alpha}$ set of a fuzzy real number $X$ for $0<\alpha \leq 1$, defined as $X^{\alpha}=\{t \in R: X(t) \geq \alpha\}$.

A fuzzy real number $X$ is called convex, if $X(t) \geq X(s) \wedge X(r)=$ $\min (X(s), X(r))$, where $s<t<r$.

If there exists $t_{0} \in R$ such that $X\left(t_{0}\right)=1$, then the fuzzy real number $X$ is called normal. A fuzzy real number $X$ is said to be upper semi- continuous if for each $\varepsilon>0, X^{-1}([0, a+\varepsilon))$, for all $a \in L$ is open in the usual topology 
of $R$. The set of all upper semi-continuous, normal, convex fuzzy number is denoted by $L(R)$.

The absolute value $|X|$ of $X \in L(R)$ is defined as (see for instance Kaleva and Seikkala [2])

$$
|X|(t)= \begin{cases}\max \{X(t), X(-t)\}, & \text { if } \quad t \geq 0 \\ 0, & t<0\end{cases}
$$

Let $\bar{d}: L(R) \times L(R) \rightarrow R$ be defined by

$$
\bar{d}(X, Y)=\sup _{0 \leq \alpha \leq 1} d\left(X^{\alpha}, Y^{\alpha}\right)
$$

Then $\bar{d}$ defines a metric on $L(R)$.

A sequence $X=\left(X_{k}\right)$ of fuzzy numbers is a function $X$ from the set $N$ of all positive integers into $L(R)$. The fuzzy number $X_{k}$ denotes the value of the function at $k \in N$ and is called the $k$-th term or general term of the sequence. The set of all sequences of fuzzy numbers is denoted by .

A sequence $\left(X_{k}\right)$ of fuzzy real numbers is said to be convergent to the fuzzy real number, if for every $\varepsilon>0$, there exists $k_{0} \in N$ such that $\bar{d}\left(X_{k}, X_{0}\right)<\varepsilon$ for all $k \geq k_{0}$.

A sequence $X=\left(X_{k}\right)$ of fuzzy numbers is said to be $I$-convergent if there exists a fuzzy number $X_{0}$ such that for all $\varepsilon>0$, the set $\{k \in N$ : $\left.\bar{d}\left(X_{k}, X_{0}\right) \geq \varepsilon\right\} \in I$. We write $I-\lim X_{k}=X_{0}$.

A sequence $\left(X_{k}\right)$ of fuzzy numbers is said to be $I$-bounded if there exists a real number $\mu$ such that the set $\left\{k \in N: \bar{d}\left(X_{k}, \overline{0}\right)>\mu\right\} \in I$.

If $I=I_{f}$, then $I_{f}$ convergence coincides with the usual convergence of fuzzy sequences. If $I=I_{d}\left(I_{\delta}\right)$, then $I_{d}\left(I_{\delta}\right)$ convergence coincides with statistical convergence (logarithmic convergence) of fuzzy sequences. If $I=$ $I_{u}, I_{u}$ convergence is said to be uniform convergence of fuzzy sequences.

A double sequence of fuzzy real numbers is a double infinite array of fuzzy real numbers. We denote a double sequence of fuzzy real numbers by $\left(X_{k, l}\right)$, where $\left(X k, l^{\prime} s\right.$ are fuzzy real numbers for each $k, l \in N$. Throughout the article ${ }_{2} w^{F}$ denote the set of all double sequences of fuzzy real numbers.

A double sequence $\left(X_{k, l}\right)$ of fuzzy numbers is said to be convergent in Pringsheim sence or $P$-convergent to a fuzzy real number $X_{0}$ if for each $\varepsilon>0$ there exist $k_{0}, l_{0} \in N$ such that

$$
\bar{d}\left(X_{k, l}, X_{0}\right)>\varepsilon \text { for all } k \geq k_{0}, l \geq l_{0} \text {. We write } P-\lim x_{k, l}=X_{0} .
$$

A double sequence $\left(X_{k, l}\right)$ of fuzzy numbers is said to be null in Pringsheim sence or $P$-null if $P-\lim X_{k, l}=\overline{0}$.

A double sequence $\left(X_{k, l}\right)$ of fuzzy numbers is said to be bounded in Pringsheim sence or $P$-bounded if $\sup _{k, l} \bar{d}\left(X_{k, l}, X_{0}\right)<\infty$. 
Let $I_{2}$ be an ideal of $2_{N \times N}$. A double sequence $\left(X_{k, l}\right)$ of fuzzy numbers is said to be $I$-convergent in Pringsheim sense if for each $\varepsilon>0$ such that

$$
\left\{(k, l) \in N \times N: \bar{d}\left(x_{k, l}, X_{0}\right) \geq \varepsilon\right\} \in I_{2}
$$

We write $I-\lim X_{k, l}=X_{0}$. For $X_{0}=\overline{0}$, it is called $I$-null in Pringsheim sence.

Let $I_{2}$ be an ideal of $2_{N \times N}$ and $I$ be an ideal of $2^{N}$. A double sequence $\left(X_{k, l}\right)$ of fuzzy numbers is said to be regularly $I$-convergent to a fuzzy number if it is $I$-convergent in Pringsheim sense and for each $\varepsilon>0$ the followings hold: For each $l \in N$ there exists $L_{l} \in L(R)$ such that $\left\{k \in N: \bar{d}\left(X_{k, l}, L_{l}\right) \geq \varepsilon\right\} \in I$, and for each $k \in N$ there exists $k \in L(R)$ such that $\left\{l \in N: \bar{d}\left(X_{k, l}, M_{K}\right) \geq \varepsilon\right\} \in I$. If $L_{l}=M_{k}=\overline{0}$ for all $l \in N$, the sequence $\left(X_{k, l}\right)$ is said to be regularly $I$-null.

A double sequence $\left(X_{k, l}\right)$ of fuzzy numbers is said to be $I$-Cauchy if for each $\varepsilon>0$ there exists $s=s(\varepsilon), t=t(\varepsilon) \in N$ such that $\{(k, l) \in N \times N$ : $\left.\bar{d}\left(X_{k, l}, X_{s, t}\right) \geq \varepsilon\right\} \in I_{2}$.

A double sequence $\left(X_{k, l}\right)$ of fuzzy numbers is said to be $I$-bounded if there exists a real number $M>0$ such that $\left\{(k, l) \in N \times N: \bar{d}\left(X_{k, l}, \overline{0}\right) \geq M\right\} \in I_{2}$.

Throughout, ${ }_{2} w^{I(F)},{ }_{2} w_{0}^{I(F)}$ and ${ }_{2} w_{\infty}^{I(F)}$ denote the spaces of fuzzy realvalued $I$-convergent, $I$-null and $I$-bounded sequences respectively.

It is clear from the definitions that ${ }_{2} w_{0}^{I}(F) \subset_{2} w^{I(F)} \subset_{2} w_{\infty}^{I(F)}$ and the inclusions are proper.

It can be easily shown that ${ }_{2} w_{\infty}^{I(F)}$ is complete with respect to the metric $f$ defined by $f(X, Y)=\sup _{k, l} \bar{d}\left(X_{k, l}, Y_{k, l}\right)$, where $X=\left(X_{k, l}\right), Y=\left(Y_{k, l}\right) \in$ ${ }_{2} w_{\infty}^{I(F)}$.

Lemma 1. Let $\left(\alpha_{K}\right)$ and $\left(\beta_{k}\right)$ be sequences of real or complex numbers and $\left(p_{k}\right)$ be a bounded sequence of positive real numbers, then

$$
\left|\alpha_{k}+\beta_{k}\right|^{p_{k}} \leq D\left(\left|\alpha_{k}\right|^{p_{k}}+\left|\beta_{k}\right|^{p_{k}}\right)
$$

and

$$
|\lambda|^{p_{k}} \leq \max \left(1,|\lambda|^{H}\right)
$$

where $D=\max \left(1,|\lambda|^{H-1}, H=\sup p_{k}, \lambda\right.$ is any real or complex number.

Lemma 2. If $\bar{d}$ is translation invariant then

(a) $\bar{d}\left(X_{k, l}+Y_{k, l}, 0\right) \leq \bar{d}\left(X_{k, l}, 0\right)+\bar{d}\left(Y_{k, l}, 0\right)$

(b) $\bar{d}\left(\alpha X_{k, l}, 0\right) \leq|\alpha| \bar{d}\left(X_{k, l}, 0\right),|\alpha|>1$.

Let $f$ be a double sequence of modulus functions, $p=\left(p_{k, l}\right)$ be a bounded double sequence of strictly positive real numbers. We define the following new sequence spaces as:

$$
\begin{aligned}
{ }_{2} w^{I(F)}(f, p)= & \left\{X=\left(X_{k, l}\right) \in \in_{2} w^{F}: I-\lim f\left(\left[\bar{d}\left(X_{k, l}, X_{0}\right)\right]^{p}\right)=0,\right. \\
& \text { for } \left.X_{0} \in L(R)\right\} \in I_{2},
\end{aligned}
$$




$$
\begin{gathered}
{ }_{2} w_{0}^{I(F)}(f, p)=\left\{X=\left(X_{k, l}\right) \in \in_{2} w^{F}: I-\lim f\left(\left[\bar{d}\left(X_{k, l}, \overline{0}\right)\right]^{p}\right)=0\right\} \in I_{2}, \\
{ }_{2} w_{\infty}^{I(F)}(f, p)=\left\{X=\left(X_{k, l}\right) \in_{2} w^{F}: I-\sup _{k, l} f\left(\left[\bar{d}\left(X_{k, l}, \overline{0}\right)\right]^{p}\right)<\infty\right\} \in I_{2} .
\end{gathered}
$$

\section{Some special cases:}

a. If $F(x)=x$, then the above spaces becomes,

$$
\begin{aligned}
{ }_{2} w^{I(F)}(p)= & \left\{X=\left(X_{k, l}\right) \in \in_{2} w^{F}: I-\lim \left[\bar{d}\left(X_{k, l}, X_{0}\right)\right]^{p}=0,\right. \\
& \text { for } \left.X_{0} \in L(R)\right\} \in I_{2}, \\
{ }_{2} w_{0}^{I(F)}(p)=\left\{X=\left(X_{k, l}\right) \in_{2} w^{F}: I-\lim \left[\bar{d}\left(X_{k, l}, \overline{0}\right)\right]^{p}=0\right\} \in I_{2}, & \left\{X_{k, l}\left[X_{\infty}^{I(F)}(p)=\left\{X=\left(X_{k, l}\right) \in_{2} w^{F}: I-\sup _{k}\left[\bar{d}\left(X_{k, l}, \overline{0}\right)\right]^{p}\right)<\infty\right\} \in I_{2} .\right.
\end{aligned}
$$

b. If $\left(p_{k, l}\right)=1$ for all $k, l \in N$, we have,

$$
\begin{aligned}
&{ }_{2} w^{I(F)}(f)=\left\{X=\left(X_{k, l}\right) \in \in_{2} w^{F}: I-\lim f\left(\left[\bar{d}\left(X_{k, l}, X_{0}\right)\right]\right)=0,\right. \\
&\text { for } \left.X_{0} \in L(R)\right\} \in I_{2}, \\
&{ }_{2} w_{0}^{I(F)}(f)=\left\{X=\left(X_{k, l}\right) \in \in_{2} w^{F}: I-\lim f\left(\left[\bar{d}\left(X_{k, l}, \overline{0}\right)\right]\right)=0\right\} \in I_{2}, \\
&{ }_{2} w_{\infty}^{I(F)}(f)=\left\{X=\left(X_{k, l}\right) \in_{2} w^{F}: I-\sup _{k, l} f\left(\left[\bar{d}\left(X_{k, l}, \overline{0}\right)\right]\right)<\infty\right\} \in I_{2} .
\end{aligned}
$$

c. If $f(x)=x$ and $\left(p_{k, l}\right)=1$ for all $k, l \in N$, then

$$
\begin{aligned}
{ }_{2} w^{I(F)}= & \left\{X=\left(X_{k, l}\right) \in \epsilon_{2} w^{F}: I-\lim \left[\bar{d}\left(X_{k, l}, X_{0}\right)\right]=0,\right. \\
& \text { for } \left.X_{0} \in L(R)\right\} \in I_{2}, \\
{ }_{2} w_{0}^{I(F)}= & \left\{X=\left(X_{k, l}\right) \in_{2} w^{F}: I-\lim \left[\bar{d}\left(X_{k, l}, \overline{0}\right)\right]=0\right\} \in I_{2}, \\
{ }_{2} w_{\infty}^{I(F)}= & \left\{X=\left(X_{k, l}\right) \in_{2} w^{F}: I-\sup _{k, l}\left[\bar{d}\left(X_{k, l}, \overline{0}\right)\right]<\infty\right\} \in I_{2} .
\end{aligned}
$$

\section{Main results}

Theorem 1. Let $f$ be a modulus function, then ${ }_{2} w^{I(F)}(f, p),{ }_{2} w_{0}^{I(F)}(f, p)$ and ${ }_{2} w_{\infty}^{I(F)}(f, p)$ are linear spaces.

Proof. We will prove the result for ${ }_{2} w_{0}^{I(f)}(f, p)$, others are same. Let, $X=\left(X_{k, l}\right)$ and $Y=\left(Y_{k, l}\right) \in{ }_{2} w_{0}^{I(F)}(f, p)$. For scalars $\alpha, \beta \in C$, there exist integers $a_{\alpha}$ and $b_{\beta}$ such that $|\alpha| \leq a_{\alpha}$ and $|\beta| \leq b_{\beta}$. Since $f$ be a modulus function, we have

$$
\begin{aligned}
f\left(\left[\bar{d}\left(\left(\alpha X_{k}+\beta Y_{k}\right), \overline{0}\right)\right]^{p}\right) \leq & D\left(a_{\alpha}\right)^{H} f\left(\left[\bar{d}\left(X_{k, l}, \overline{0} 0\right]^{p}\right)\right. \\
& +D\left(b_{\beta}\right)^{H} f\left(\left[\bar{d}\left(Y_{k, l}, \overline{0}\right)\right]^{p}\right) \rightarrow 0 \text { as } k, l \rightarrow \infty .
\end{aligned}
$$


Therefore, $\alpha X_{k}+\beta Y_{k} \in{ }_{2} w_{0}^{I(F)}(F, p)$. This completes the proof.

Theorem 2. Let $f$ be a modulus function, then ${ }_{2} w_{\infty}^{I(F)}(p) \subset{ }_{2} w_{\infty}^{I(F)}(f, p)$.

Proof. Let $X=\left(X_{k, l}\right) \in{ }_{2} w_{\infty}^{I(F)}(p)$, then we have $I-\sup _{k, l} f\left(\left[\bar{d}\left(X_{k, l}, \overline{0}\right)\right]^{p}\right)$ $<\infty$. Let $\varepsilon>0$ and choose a $\delta>0$ with $0<\delta<1$ such that $f(t)<\varepsilon$ for $0 \leq \delta \leq 1$. Thus

$$
\begin{aligned}
I-\sup _{k, l} f\left(\left[\bar{d}\left(X_{k, l}, \overline{0}\right)\right]^{p}\right)= & I-\sup _{k, l, \bar{d}\left(X_{k, l}, \overline{0} \leq \delta\right.} f\left(\left[\bar{d}\left(X_{k, l}, \overline{0}\right)\right]^{p}\right) \\
& +I-\sup _{k, l, \bar{d}\left(X_{k, l}, \overline{0}>\delta\right.} f\left(\left[\bar{d}\left(X_{k, l}, \overline{0}\right)\right]^{p}\right) \\
& \leq \varepsilon+\frac{M}{\delta} \sup _{k, l}\left[\left(\bar{d}\left(x_{k, l}, \overline{0}\right)^{p}\right]\right. \\
& <\infty \text { by properties of modulus function. }
\end{aligned}
$$

Hence $X=\left(X_{k, l}\right) \in{ }_{2} w_{\infty}^{I(F)}(f, p)$. This completes the proof.

Theorem 3. Let $f$ be a modulus function and $\alpha=\lim _{t \rightarrow \infty} \frac{f(t)}{t}>0$, then ${ }_{2} w_{\infty}^{I(F)}(F, p) \subset{ }_{2} w_{\infty}^{I(F)}(p)$.

Proof. Let $X=\left(X_{k, l}\right) \in{ }_{2} w_{\infty}^{I(F)}(f, p)$. By definition of $\alpha$, we have $f(t) \geq \alpha t$ for all $t \geq 0$. Since, $\alpha>0$, we have $t \leq \frac{f(t)}{\alpha}$. Thus,

$$
I-\sup _{k, l}\left(\left[\bar{d}\left(X_{k, l}, \overline{0}\right)\right]^{p}\right) \leq I-\frac{1}{\alpha} \sup _{k, l} f\left(\left[\bar{d}\left(X_{k, l}, \overline{0}\right)\right]^{p}\right)<\infty .
$$

This follows that $X=\left(X_{k, l}\right) \in{ }_{2} w_{\infty}^{I(F)}(p)$.

Theorem 4. Let $f$ be a modulus function then ${ }_{2} w_{\infty}^{I(F)} \subset{ }_{2} w_{0}^{I(F)}(f, p)$, if $\lim _{t \rightarrow \infty} f(t)=0$ for $t>0$.

Proof. It is easy to prove, so omitted.

Theorem 5. Let $f$ be a modulus function and if $\lim _{t \rightarrow \infty} f(t)=\infty$ for $t>0$ then ${ }_{2} w_{\infty}^{I(F)}(f, p) \subset{ }_{2} w_{0}^{I(F)}$. Then,

Proof. Let $\lim _{t \rightarrow \infty} f(t)=\infty$ for $t>0$. If $X=\left(X_{k, l}\right) \in{ }_{2} w_{\infty}^{I(F)}(f, p)$.

$$
f\left(\left[\bar{d}\left(X_{k, l}, \overline{0}\right)\right]^{p}\right) \leq M<\infty \text { for all } k, l .
$$

If possible let $X=\left(X_{k}\right) \notin{ }_{2} w_{0}^{I(F)}$, then for some $\varepsilon>0$ there exists a positive integer $k_{0}$ such that $\bar{d}\left(X_{k, l}, \overline{0}\right)<\varepsilon$ for $k \geq k_{0}, l \geq l_{0}$. Therefore,

$$
f(\varepsilon) \geq f\left(\left[\bar{d}\left(x_{k, l}, \overline{0}\right)\right]^{p}\right) \leq M \text { for } k \geq k_{0}, l \geq l_{0} .
$$


This contradicts to our assumption that $\lim _{t \rightarrow \infty} f(t)=\infty$ for $t>0$ and hence $X=\left(X_{k, l}\right) \in{ }_{2} w_{0}^{I(F)}$ and completes the proof.

Theorem 6. If $f$ be a modulus function, then ${ }_{2} w_{0}^{I(F)}(f, p)$ and ${ }_{2} w_{\infty}^{I(F)}(f, p)$ are paranormed spaces with the paranorm $h$ defined by

$$
h(X)=\sup _{k, l}\left\{f\left[\bar{d}\left(X_{k, l}, \overline{0}\right)\right]^{p}\right\}^{\frac{1}{M}}
$$

where $M=\max \left\{1 \sup _{k, l} p\right\}$.

Proof. Obviously $h(X)=h(-X)$ for all $x \in{ }_{2} w_{0}^{I(F)}(f, p)$. It is trivial that $X_{k, l}=\overline{0}$ for $X=\overline{0}$. Since, $\frac{p}{M} \leq 1$, since $\bar{d}$ is translation invariant and by using Minkowski's inequality, we have,

$$
\left\{f\left[\bar{d}\left(\left(X_{k, l}+Y_{k, l}\right), \overline{0}\right)\right]^{p}\right\}^{\frac{1}{M}} \leq\left\{f\left[\bar{d}\left(\left(X_{k, l}, \overline{0}\right)\right]^{p}\right\}^{\frac{1}{M}}+\left\{f\left[\bar{d}\left(\left(Y_{k, l}, \overline{0}\right)\right]^{p}\right\}^{\frac{1}{M}} .\right.\right.
$$

Hence,

$$
h(X+Y) \leq h(X)+h(Y) .
$$

Finally to check the continuity of scalar multiplication, let be any scalar, by definition we have

$$
h(\lambda X)=\sup _{k, l}\left\{f\left[\bar{d}\left(\lambda X_{k, l}, \overline{0}\right)\right]^{p}\right\}^{\frac{1}{M}} \leq K_{\lambda}^{\frac{H}{M}} h(X),
$$

where $H=\sup _{k, l} p<\infty$. Where $K_{\lambda}$ is positive integer such that $|\lambda| \leq K_{\lambda}$. Let $\lambda \rightarrow 0$ for any fixed $X$ with $h(X)=0$. By definition for $|\lambda| \leq 1$, we have

$$
\sup _{k, l}\left\{f \left[\bar{d}\left(\lambda X_{k, l}, \overline{0}^{p}\right\} \leq \varepsilon \text { for } N>N(\varepsilon) .\right.\right.
$$

Also for $1 \leq n \leq N$ by taking $\lambda$ small enough, since $f$ is continuous, we get

$$
\sup _{k, l}\left\{f \left[\bar{d}\left(\lambda X_{k, l}, \overline{0}^{p}\right\} \leq \varepsilon .\right.\right.
$$

Implies that $h(\lambda X) \rightarrow 0$ as $\lambda \rightarrow 0$. This completes the proof.

Theorem 7. If $I$ is an admissible ideal then the spaces ${ }_{2} w^{I(F)}(f, p)$, ${ }_{2} w_{0}^{I(F)}(f, p)$ and ${ }_{2} w_{\infty}^{I(F)}(f, p)$ are complete metric spaces under the metric

$$
h(X, Y)=\sup _{k}\left\{f\left[\bar{d}\left(X_{k, l}, Y_{k, l}\right)\right]^{p}\right\}^{\frac{1}{M}},
$$

where $M=\max \left\{1, \sup _{k, l} p\right\}$. 
Proof. It is easy to see that $h$ is a metric on ${ }_{2} w^{I(F)}(f, p)$. To show completeness. Let $\left(X^{i}\right)$ be a Cauchy sequence in ${ }_{2} w^{I(F)}(f, p)$ where $\left(X^{i}\right)=$ $\left(X_{k, l}^{i}\right)$. Therefore for each $\varepsilon>0$ there exists $i_{0} \in N$ such that

$$
h\left(X^{i}, X^{j}\right)<\varepsilon \text { for all } i, j \geq i_{0}
$$

i.e

$$
\sup _{k, l}\left(f\left[\bar{d}\left(X_{k, l}^{i}, Y_{k, l}^{j}\right)\right]^{p}\right)^{\frac{1}{M}}<\varepsilon \text { for all } i, j \geq i_{0} .
$$

This means

$$
\sup _{k, l}\left\{f\left[\bar{d}\left(X_{k, l}^{i}, Y_{k, l}^{j}\right)\right]^{p}\right\}^{\frac{1}{M}}<\varepsilon \text { for all } i, j \geq i_{0} .
$$

Since $f$ is modulus function, so choosing suitable $\varepsilon_{1}>0$ and we obtain

$$
\left.\bar{d}\left(X_{k, l}^{i}, Y_{k, l}^{j}\right)\right]<\varepsilon_{1} \text { for all and for each } k, l
$$

i.e

$$
\left(X_{k}^{i}\right) \text { is a Cauchy sequence in } L(R) \text { for each } k, l \text {. }
$$

Keeping fixed and letting $j \rightarrow \infty$, one can find that

$$
\left.\sup _{k, l}\left(f\left[\bar{d}_{k, l}^{i}, X_{k, l}\right)\right]^{p}\right)<\varepsilon \text { for all } i \geq i_{0} .
$$

That means,

$$
h\left(X^{i}, X\right)<\varepsilon \text { for all } i \geq i_{0} .
$$

Next to show $X \in_{2} w^{I(F)}(f, p)$, for which the proof as follows:

Since for $\left(X_{k, l}^{i}\right) \in_{2} w^{I(F)}(f, p)$, so for $i, j$, there exist $L^{i}, L^{j} \in L(R)$ and $k_{i}, k_{j} i \in N$ and $l_{i}, l_{j} \in N$, such that

$$
\sup _{k, l}\left(f\left[\bar{d}\left(X_{k, l}^{i}, L^{i}\right)\right]^{p}\right)<\varepsilon \text { for all } k \geq k_{i}, l \geq l_{i} .
$$

and

$$
\sup _{k, l}\left(f\left[\bar{d}\left(X_{k, l}^{i}, L^{i}\right)\right]_{k}^{p}\right)<\varepsilon \text { for all } k \geq k_{j}, l \geq l_{j} .
$$

Now let $k_{0}=\max \left(k_{i}, k_{j}\right)$ and $l_{0}=\max \left(l_{i}, l_{j}\right)$; for $i, j \geq i_{0}$, we have

$$
\begin{aligned}
\sup \left(f\left[\bar{d}\left(\left(L^{i}, L^{j}\right)\right]^{p}\right) \leq\right. & C \sup _{k, l}\left(f\left[\bar{d}\left(L^{i}, X_{k, l}^{i}\right)\right]^{p}\right) \\
& +C \sup _{k, l}\left(f\left[\bar{d}\left(X_{k, l}^{i}, X_{k, l}^{j}\right)\right]^{p}\right)+C \sup _{k, l}\left(f\left[\bar{d}\left(X_{k, l}^{j}, L^{j}\right)\right]^{p}\right) \\
< & 3 C \varepsilon \text { for all } i, j \geq i_{0} \text { and } k \geq k_{0}, l \geq l_{0} .
\end{aligned}
$$


Hence $\left(L^{i}\right)$ is a Cauchy sequence in $L(R)$. So there exists $L \in L(R)$ such that $L^{i} \rightarrow L$ as $i \rightarrow \infty$. Now keeping fixed and letting $j \rightarrow \infty$,once can find that,

$$
\sup \left(f\left[\bar{d}\left(L^{i}, L\right)\right]^{p}\right)<3 C \varepsilon \text { for all } i \geq i_{0}
$$

Therefore,

$$
\begin{aligned}
\sup _{k, l}\left(f\left[\bar{d}\left(X_{k, l}, L\right)\right]^{p}\right) \leq & C \sup _{k, l}\left(f\left[\bar{d}\left(X_{k, l}, X_{k, l}^{i_{0}}\right]^{p}\right)\right. \\
& +\sup _{k, l}\left(f\left[\bar{d}\left(X_{k, l}^{i}, L^{i}\right)\right]^{p}\right)+\sup \left(f\left[\bar{d}\left(L^{i_{0}}, L\right)\right]^{p}\right) \\
< & 2 C \varepsilon+3 C^{2} \varepsilon \cong \varepsilon_{1} \text { for all } k \geq k_{0}, l \geq l_{0} .
\end{aligned}
$$

This implies that $X=\left(X_{k, l}\right) \in{ }_{2} w^{I(F)}(f, p)$. This completes the proof.

\section{References}

[1] Fridy J.A., On statistical convergence, Analysis, 5(1985), 301-313.

[2] Kaleva O., Seikkala S., On fuzzy metric spaces, Fuzzy Sets and Systems, 12(1984), 215-229.

[3] Kamthan P.K., Gupta M., Sequence Spaces and Series, Marcel Dekkar, 1980.

[4] Kostyrko P., Šalát T., Wilczyńki W., I-convergence, Real Anal. Exchange, 26(2000-2001), 669-686.

[5] Kizmaz H., On certain sequence spaces, Canad. Math. Bull., 24(2)(1981), 169-176.

[6] Krasnoselkit M.A., Rutitsky Y.B., Convex functions and Orlicz functions, Groningen, Netherlands, 1961.

[7] Lindenstrauss J., Tzafriri L., On Orlicz sequence spaces, Israel J. Math., 101(1971), 379-390.

[8] Matloka M., Sequences of fuzzy numbers, BUSEFAL, 28(1986), 28-37.

[9] Nakano H., Modular sequence spaces, Proc. Japan Acad., 27(1951), 508-512.

[10] Šalát T., Tripathy B.C., Ziman M., On some properties of I-convergence, Tatra Mountain Publ. Math., 28(2004), 279-286.

[11] Sarabadan S., Talebi S., On I-convergence of double sequences in 2-normed spaces, Int. J. Contemp. Math. Sciences, 7(14)(2012), 673-684.

[12] Tripathy B.K., Tripathy B.C., On I-convergent double sequences, Soochow Jour. Math., 31(4)(2005), 549-560.

[13] Tripathy B.C., Sarma B., Statistically convergent double sequence spaces defined by Orlicz function, Soochow Journal of Mathematics, 32(2)(2006), 211-221.

[14] Tripathy B.C., Sarma B., Some double sequence spaces of fuzzy numbers defined by Orlicz functions, Acta Math. Scientia, Ser. B, 31(1)(2011), 134-140.

[15] Tripathy B.C., Das P.C., Some classes of difference sequences of fuzzy real numbers, Fasc. Math., 40(2008), 105-117.

[16] ZadeH L.A., Fuzzy sets, Inform and Control, 8(1965), 338-353. 
[17] Zygumd A., Trigonometric Series, vol. II, Cambridge, 1993.

\author{
MANMOHAN DAS \\ Department of MATHEMAtics \\ Bajali College(Gauhati University) \\ Assam, India \\ e-mail: mdas.bajali@gmail.com
}

Received on 11.07.2014 and, in revised form, on 03.07.2015. 